\section{Superconductivity and Liquid Helium II}

WHEN discussing our observations on the transfer of liquid helium II along solid surfaces above the liquid level ${ }^{1}$, we pointed out that this mechanism of surface flow might also be responsible for the "trans. port phenomena' in the bulk liquid (high heat conduction, low viscosity and the fountain effect) ${ }^{2}$. Subsequent experiments by ourselves ${ }^{3}$, Allen and Reekie $^{4}$ and by Kapitza ${ }^{5}$ strongly support this assumption. It appears that the transfer film observed above the liquid level also extends below it, covering all solid surfaces in contact with liquid helium II, and it is evidently in this film that the anomalous transport phenomena take place.

It has been suggested by $F$. London ${ }^{6}$ that the $\lambda$-phenomenon in helium may be caused by the existence of helium atoms of very low (or zero) thermal energy, and the mechano-caloric effect found by us ${ }^{3}$ strengthens this view. This effect shows that by flow along a solid surface, such thermally 'unexcited' atoms can be separated to a certain extent from the bulk liquid.

In elaborating our hypothesis we have now come across a striking analogy between the phenomenology of liquid helium II and superconductivity, which seems to go much farther than a superficial similarity. While we have a good deal of information on the superconductive state, our knowledge concerning liquid helium II is very limited. A certain amount of generalization has thus been necessary in the formulation of our thesis, which therefore must be considered as of an approximate nature only. The main points of the analogy are as follows :

(1) As it is impossible to establish a difference of electrical potential at the ends of a superconductor, so it is impossible to establish a temperature difference along the transfer film of helium II.

(2) In both cases the frictionless transport of particles (electrons or atoms) breaks down as soon as a certain value of flow is exceeded. This limiting value is given by the current threshold in superconductors, $J$, and by the rate of transfer, $R$, in helium ${ }^{1}$.

(3) In both cases this limiting value of flow is solely dependent on temperature and is independent of the length of the path the particles have to travel. It is directly proportional to the width of the connecting surface (this holds rigidly for plane surfaces, whereas for curved surfaces the limiting rate of flow will be reduced in both cases).

(4) In liquid helium as well as in superconductors, the frictionless transport seems to be confined to the geometricai surface of the substance. Kapitza ${ }^{5}$ has demonstrated that an anomalously high heat conduction does not take place in the bulk of helium II ; that is, there is no frictionless transport of atoms unless this can take place along a solid - liquid boundary surface. An analogy to this exists in a superconductor, since it seems theoretically impossible to remove an electric charge from inside a completely closed superconducting shell except by the process of normal conduction.

(5) It appears that in both cases the maximum current density of flow on the surface $(J, R)$ indicates the number of particles which can at each tempera. ture be transported frictionlessly (provided one assumes the particles to have velocities independent of temperature). The threshold curve and the curve giving the temperature dependency of $R$ will therefore play an identical part in the treatment of both phenomena. Indeed the shape of these curves is similar; they both start with a finite slope at the temperature at which the first anomalous behaviour is evident, and they both become temperature independent near absolute zero.

(6) When developing the thermodynamies of a superconductor with the current density rather than the magnetic field as the variable of state, one arrives at a formalism which also fits well the observations in liquid helium II. While the rigorous derivation of this cannot be given here, it appears that $R^{2}$ assumes the same significance in determining the free energy of liquid helium II as the square of the threshold field has for superconductors?.

(7) One would expect that in such a model the specific heat of the system of superconductive electrons as well as that of the 'unexcited' helium atoms would be zero. This seems actually to be the case. The facts that a super-current in a temperature gradient produces no Thomson heat ${ }^{8}$ and that no heat transport exists in a helium film in a direction opposite to that of the flow ${ }^{1}$ strongly suggest that no heat is transferred to the particles while they remain in the anomalous state. We ascribe the observed high specific heat of superconductive metals and liquid helium II to the energy taken up in lifting particles from the lowest state into thermal equilibrium with the rest of the substance.

The question naturally arises what physical significance has to be given to this striking analogy. It seems to us that the fundamental phenomenon is the passing at finite temperatures of a number of particles into the lowest quantum state, where no energy exchange can take place between them and the remainder of the substance. This new form of aggregation of matter evidently follows quite general rules, so that the fact that the particles are electrons in one case and atoms in the other is only of secondary importance. The phenomenon of frictionless transport confined, as it is in both cases, to the geometrical surface, might be explained by the fact that, as the particles are free from interaction with the bulk of the substance, they can only be accelerated by external forces. The range to which these external forces can penetrate the substance limits the layer in which the transport takes place. Thus, in a superconductor, electrons can only be moved within the depth of penetration of an external magnetic field", while the flow in a helium film is limited by the extent of the van der Waals' forces of the container. It appears from these considerations that the theoretical interpretations of the superconductive state and of the $\lambda$-phenomenon in liquid helium II should be similar in their fundamental aspect, and it is with regard to such a generalized theoretical treatment that we hope the analogy pointed out in this note will be of value.

Clarendon Laboratory, $\quad$ K. Mendelssohn. Oxford

Nov. 5.

${ }^{1}$ Daunt and Mendelssohn, Proc. Roy. Soc., A, 170, 423 (1939).

- See, for example, Darrow, Rev. Mod. Phys., 12, 257 (1940), and Jones, "Reports on Progress in Physics", 8,280 (1940).

'Dannt and Mendelssohn, NATuRE, 143, 719 (1939).

- Allen and Reekie, NATURE, 144, 475 (1939).

${ }^{8}$ Kapitza, J. Phys. U.S.S.R., 1, 181 (1941).

'London, F., Phys. Rev., 54, 947 (1938).

7 Gorter and Casimir, Physicx, 1, 305 (1934).

- Daunt and Mendelssohn, Nature, 141, 116 (1938).

'London, H., Proc. Roy. Soc., A, 152, 850 (1935). 